\title{
Ternary copper(II)-polypyridyl enantiomers: aldol-type condensation, characterization, DNA-binding recognition, BSA-binding and anticancer property.
}

\begin{abstract}
Chiral enantiomers $[\mathrm{Cu}($ phen $)(\mathrm{l}$-threo $)(\mathrm{H} 2 \mathrm{O})] \mathrm{NO} 31$ and $[\mathrm{Cu}($ phen $)(\mathrm{d}-\mathrm{threo})(\mathrm{H} 2 \mathrm{O})] \mathrm{NO} 32$ (threo $=$ threoninate) underwent aldol-type condensation with formaldehyde, with retention of chirality, to yield their respective enantiomeric ternary copper(ii) complexes, viz.l- and d$[\mathrm{Cu}$ (phen)(5MeOCA)(H2O)]NO3.xH $2 \mathrm{O}$ (3 and 4; phen = 1,10-phenanthroline; 5MeOCA = 5-methyloxazolidine-4-carboxylate; $x=0$-3) respectively. These chiral complexes were characterized by FTIR, elemental analysis, circular dichroism, UV-Visible spectroscopy, fluorescence spectroscopy (FL), molar conductivity measurement, ESI-MS and X-ray crystallography. Analysis of restriction enzyme inhibition by these four complexes revealed modulation of DNA binding selectivity by the type of ligand, ligand modification and chirality. Their interaction with bovine serum albumin was investigated by FL and electronic spectroscopy. With the aid of the crystal structure of BSA, spectroscopic evidence suggested their binding at the cavity containing Trp134 with numerous Tyr residues in subdomain IA. The products were more antiproliferative than cisplatin against cancer cell lines HK-1, MCF7, HCT116, HSC-2 and C666-1 except HL-60, and were selective towards nasopharyngeal cancer HK-1 cells over normal NP69 cells of the same organ type.
\end{abstract}

Keyword: Chiral enantiomers; Aldol-type condensation; DNA binding. 